\title{
Mecanismos de interacción entre el extracto etanólico de Jatropha curcas L. y metoclopramida en el sistema gastrointestinal
}

\author{
Sergio Goicochea-Lugo 1, 2, Ernesto Zavala-Flores 1, 2, Alberto Salazar-Granara '
}

\section{RESUMEN}

Objetivo: Determinar los mecanismos de interacción entre el extracto etanólico de J atrohpa curcas L. y la metoclopramida sobre el sistema gastrointestinal.

Material y Métodos: Se usó 30 ratones albinos machos, en5 grupos; los que recibieron por vía oral: Grupo 1: Jatropha curcas L. 800 mg/Kg, y 0, 5mg/Kg de metoclopramida. Grupo 2: 0.5 mg/mL de metoclopramida. Grupo 3: 1.5 mg/Kg de Atropina. Grupo 4: 800 mg/kg de Jatropha curcas L. Grupo 5: no recibió medicamento. A todos, se les administró por vía oral: carbón activado al 5\% 0,1 mL/10g, como marcador intestinal. Se empleó el Método Arboset al, para evaluar la motilidad intestinal. La validación estadística del recorrido intestinal se realizó aplicando las pruebas de Kolmogorov Smirnov, ANOVA de 1 cola, Tukey y Newman-Keuls.

Resultados: Se observó un el porcentaje de recorrido del carbón de $36.46 \%$ del grupo 1 frente a $65,45 \%, 3,66 \%$ y 58,87\% de los grupos 2, 3 y 4, respectivamente; y de 58,87\% del grupo 4 frente a 20,94\% del grupo 5 .

Conclusión: Se evidenció el antagonismo entre el extracto etanólico de la semilla de J atropha curcas L. con la metoclopramida, el cual se explicaría por la interacción entre el sistema colinérgico, adrenérgico, GABAérgico y de neuropéptidos sobre la glándula suprarrenal, sistema nervioso central y gastrointestinal. (Horiz Med 2014; 14(2): 27-33)

Palabras clave: Metoclopramida, Jatropha curcas L., motilidad gastrointestinal. (Fuente: DeCS BIREME).

The mechanisms of interaction between the ethanol extract of Jatrohpa curcas L. and metoclopramide on gastrointestinal system

\begin{abstract}
Objective: Determinate the mechanisms of interaction between the ethanol extract of J atrohpacurcas L. and metoclopramide on gastrointestinal system.

Material and Methods: 30 male albino mice were used, forming 5 groups and received oral medications as follows: Group 1: Jatropha curcas L. $800 \mathrm{mg} / \mathrm{kg}$, and $0.5 \mathrm{mg} / \mathrm{Kg}$ of metoclopramide. Group 2: $0.5 \mathrm{mg} / \mathrm{mL}$ of metoclopramide. Group 3: $1.5 \mathrm{mg} / \mathrm{Kg}$ of atropine. Group 4: $800 \mathrm{mg} / \mathrm{Kg}$ of Jatropha curcas L. Group 5 received no medication. All groups receivedoral activated charcoal $0.1 \mathrm{~mL} / 10 \mathrm{~g}$ as intestinal marker. The Arbos et al method was used to evaluate intestinal motility. The statistical validation of the intestine dynamics was performed using the Kolmogorov Smirnov, 1-tailed ANOVA, Tukey and Newman-Keuls.

Results: the percentage of charcoal runs in the 1 rst group was $36.46 \%$ compared to $65.45 \%$, 3.66 \% and 58.87\% for the 2 nd, 3 th and 4th groups, respectively. In the 4th group was $58,87 \%$ compared to $20.94 \%$ in the 5 th group.

Conclusion: the antagonism between the ethanol extract of the seeds of J .curcas L. with metoclopramide, which is probably would explain by the interaction between the cholinergic system, adrenergic system, GABAergic system and neuropeptides on the adrenal gland, central nervous system and gastrointestinal system. (Horiz Med 2014; 14(2): 27-33)
\end{abstract}

Key words: metoclopramide, J atrophacurcas, gastrointestinal motility (Source: MeSH NLM).

Centro de Investigación de Medicina Tradicional y Farmacología (CIMTFAR), FMH - USMP.

2 Estudiante de medicina Humana - Sociedad Científica de Estudiantes de Medicina, USMP. (SOCIEM-USMP), Lima, Perú. 


\section{INTRODUCCIÓN}

En distintas zonas del Perú:Lima, Piura, Cajamarca, San Martín, Cuzco, Ucayali y Loreto, se relata el uso medicinal del J atropha Cucas L; como: purgante, antirreumático, analgésico, antiulceroso, uso tópico en conjuntivitis, entre otros (1).

Si bien es cierto; empíricamente, se evidencia un efecto beneficioso de la planta J.curcas L, sobre distintos problemas de la salud, algunas investigaciones pre-clínicas han demostrado su actividad anti-fértil $(2,3)$, cicatrizante, abortiva, coagulante, anticoagulante y letalidad (4-7).

Los efectos biológicos reconocidos de la semilla de J atropha curcas L, son evidenciados por la presencia de sus metabolitos secundarios. Hoy, se reconoce presencia de alcaloides, flavonoides, esteres, lectinas, entre otros (8).

El uso concomitante de plantas medicinales con fármacos, puede desencadenar reacciones adversas como por ejemplo, el aumento de riesgo de rechazo a trasplantes tras la ingesta de Hypericum perforatum L. (Hierba de San J uan) y ciclosporina (9), así como efectos sobre el International Normalized Ratio(INR), generalmente prolongándolo, entre el uso de warfarina y Panaxquinquefolium(Ginseng) (10).

El objetivo del estudio fue evaluar la interacción farmacológica sobre la motilidad intestinal del extracto etanólico de la semilla de J atropha curcas L. y la metoclopramida.

\section{MATERIAL Y MÉTODOS}

Estudio cuasi-experimental, pre-clínico, prospectivo y doble ciego; realizado en el Centro de Investigación de Medicina Tradicional y Farmacología, de la Facultad de Medicina Humana, de la Universidad de San Martín de Porres (FMHUSMP); durante el periodo Julio a Noviembre del 2013.
Se trabajó con semillas secas de Jatropha curcas L, recolectadas en Tarapoto - San Martín. La certificación taxonómica se realizó bajo los criterios del método de Cerrate, E. 1969 (11).

La población fue de 30 ratones albinos machos, cuyos pesos oscilaron entre 25 y 30 gramos. Los que se adquirieron en el Instituto Nacional de Salud (INS - Bioterio, Chorrillos, Lima - Perú); todos tuvieron un proceso de aclimatación en las instalaciones del Bioterio de la FMH-USMP, en condiciones estándares de temperatura: $22^{\circ} \mathrm{C}(+/-2)$, humedad relativa promedio entre 45 a $70 \%$, y niveles de ruido menores a $70 \mathrm{~dB}$. Para la distribución y asignación randomizada de los grupos experimentales, se empleó el método por sorteo (12).

Muestra química.- Metoclopramida, ampollas de $10 \mathrm{mg} / 2 \mathrm{~mL}$, lote EL-395038 RS EJE-5610, vencimiento 12/2013; Atropina, ampollas de 25 $\mathrm{mg} / 1 \mathrm{~mL}$, lote $101010 \mathrm{RS} \mathrm{NG}-3587$, vencimiento 01/ 2014; Carbón Activado, lote 0000097644.

Preparación del Extracto Etanólico.- Se realizó a partir de $20 \mathrm{~g}$ de semillas molidas de J atropha curcas L. maceradas en etanol al $70 \%$ durante una semana con ciclos de sacudidas de una hora. Posteriormente fue filtrado y los residuos fueron secados en una estufa por 5 días. Luego se extrajeron muestras mediante raspado, se almacenaron en envases herméticos y se refrigeraron. Las soluciones administradas tuvieron una concentración del $20 \%$, las que se prepararon de la siguiente forma: se introdujo la muestra en un recipiente, se agregó $2 \mathrm{~mL}$ de etanol al 70\% y 3 $\mathrm{mL}$ de cloroformo GP, y fue diluida en una plancha con agitador a una temperatura de $37^{\circ} \mathrm{C}$ durante 3 minutos. Finalmente se agregó agua destilada hasta completar los $100 \mathrm{~mL}$ de solución.

Esta solución, permitió, la aplicación de volúmenes adecuados de las sustancias problemas, en base a las recomendaciones estándares recopiladas del Handbook of laboratory animal science(13).

Evaluación de la motilidad gastrointestinal in vivo.Se utilizó el método de Arbos et al. 1993 (14). 24 horas antes del inicio del experimento, se privó de alimentos a los roedores, y se continuó con agua 
ad libitum. Posteriormente, se administraron las sustancias en estudio, por la vía probable. Transcurridos 30 minutos, se procedió a administrar por vía oral (VO) el marcador carbón activado al $5 \%$ a dosis de $0.1 \mathrm{~mL} / 10 \mathrm{~g}$ de peso. Luego de 30 minutos, se procedió a sacrificar a los animales por dislocación cervical. Luego, se realizó laparotomía, se extrajo el intestino delgado (desde la porción pilórica hasta el colon), y se midió la distancia recorrida del carbón activado. La distancia se expresó como la media del porcentaje de la longitud total del intestino recorrida por el carbón \pm error estándar.

Diseño de grupos experimentales.- Los grupos experimentales fueron conformados por 6 ratones, distribuidos en 5 grupos: grupo 1; de Interacción, que recibió Jatropha curcas L. $800 \mathrm{mg} / \mathrm{Kg}$, y metoclopramida a dosis establecida de $0.5 \mathrm{mg} / \mathrm{Kg}$. Grupo 2: $0.5 \mathrm{mg} /$ $\mathrm{mL}$ de metoclopramida. Grupo 3: $1.5 \mathrm{mg} / \mathrm{Kg}$ de Atropina. Grupo 4: $800 \mathrm{mg} / \mathrm{Kg}$ de Jatropha curcas L. Grupo 5: $0.1 \mathrm{ml} / 10 \mathrm{~g}$ de carbón activado.

Sistema de ciegos.- Doble ciego.En el primer sistema, dos investigadores se encargaron de

Tabla 1. Recorrido del carbón activado en los grupos experimentales la medición de la longitud total del intestino y del recorrido del carbono activado, sin tener el conocimiento del grupo al que pertenecían estas muestras. En el segundo sistema, otro investigador se encargo de procesar los datos obtenidos al software estadístico, sin conocer el grupo de origen (15).

Se consideró los estándares de manipulación de animales para trabajos de investigación, de acuerdo a: International Guiding Principles for Biomedical Research Involving Animal (1985)y el Comité de Institucional de Ética para el uso de animales en investigación - INS (16).

Para la validación estadística, se usó: test de Kolmogorov Smirnov, test de ANOVA de 1 cola, y los test de Tukey y Newman-Keuls para las comparaciones entre los grupos; se estableció la significancia estadística, para un valor $p<0.05$, y unlC al 95\%.

Se empleó el Microsoft Office Excel 2010 y el programa estadístico Graph Pad Prism Versión 5.01.

\section{RESULTADOS}

Los datos descriptivos sobre la motilidad intestinal de los grupos se presentan en la Tabla 1.

\begin{tabular}{|c|c|c|c|c|c|c|}
\hline Grupo & $\begin{array}{l}\text { Sustancia/Fármaco } \\
(\mathrm{ppm})\end{array}$ & $\mathbf{N}$ & $\begin{array}{l}\text { Media } \\
(\mathrm{cm})\end{array}$ & $\begin{array}{c}\text { Media } \\
(\%)\end{array}$ & $\begin{array}{l}\text { Desviación } \\
\text { estándar }\end{array}$ & $\begin{array}{l}\text { Test de Kolmogorov Smirnov } \\
\text { (distribución Gaussiana) }\end{array}$ \\
\hline G 1* & $\begin{array}{l}\text { Jatropha c. } 800 \mathrm{mg} / \mathrm{Kg} \\
\text { Metoclopramida }\end{array}$ & 6 & 20 & 38,46 & 17,56 & $\mathrm{Si}$ \\
\hline G2* & Metoclopramida & 6 & 40,75 & 65,45 & 7,484 & $\mathbf{S i}$ \\
\hline G3* & Atropina & 6 & 2,25 & 3,66 & 3,424 & $\mathrm{Si}$ \\
\hline G4* & Jatropha c. 800 mg/Kg & 6 & 34 & 58,87 & 23,33 & Si \\
\hline
\end{tabular}


La prueba de Kolmogorov Smirnov determinó que los grupos presentaron distribución Gaussiana o paramétrica ( $p>0,05$ IC 95\%).

La prueba de ANOVA de 1 cola mostró un valor $p<0,05$. Las pruebas de Tukey y Newman-Keuls, tuvieron un $p<0,05$ entre todos los grupos comparados, excepto en el pareo del grupo 1 versus el grupo 5 y del grupo 2 versus el grupo 4 . (Tabla 1 ).

\section{DISCUSIÓN}

La metoclopramida, fármaco utilizado en la terapéutica gastrointestinal, es un medicamento procinético cuyo mecanismo de acción esta mediado por la estimulación de receptores serotoninergicos 5-HT4, los cuales se encuentran en las células del tracto gastrointestinal, y que al ser activados, producen contracciones peristálticas. Por otro lado, también actúa a nivel central inhibiendo los receptores dopaminérgicos D2 (efecto antiemético) (21).

Diversos estudios experimentales pre clínicos, han demostrado el efecto pro cinético sobre la motilidad intestinal del extracto etanólico de J atropha curcas $L$, dato que es corroborado en el presente estudio, al presentar similitud estadística, en el recorrido en el tracto gastrointestinal, y compararlo con la metoclopramida: Tabla 1.

Estudios sobre los componentes de la planta, revelan la existencia de alcaloides como la galantamina, molécula que posee actividad anticolinesterasa (18), inductor de la liberación de serotonina, potenciador del efecto alostérico de los receptores nicotínicos y actividad sobre los receptores muscarinicos (19), Los que guardan relación con la trasmisión del efecto peristáltico del transito intestinal.

Del mismo modo, estudios sobre la fitoquimica del J atropa curcas L., evidencian la presencia de flavonoides (20). Metabolito que induce un aumento en la concentración de Adenosinamonofosfato cíclico (AMPC) al inhibir la enzima fosfodiesterasa-4 (21). Los esteres de forbol (metabolito toxico de la planta) posee una acción tipo diacilglicerol, lo que conllevaría a un incremento de las concentraciones de calcio a nivel intracelular (20).
En el presente estudio, se evidencio el antagonismo farmacológico en la interacción de J atropha curcas L. $800 \mathrm{mg} / \mathrm{Kg}$ con metoclopramida $20 \mathrm{mg} / \mathrm{Kg}$, efecto que presentó diferencias significativas con los grupos Jatropha curcas L. $800 \mathrm{mg} / \mathrm{Kg}$ y metoclopramida $20 \mathrm{mg} / \mathrm{Kg}$, lo que se observó en estudios previos, por un efecto antagónico dosis dependiente como interacción de estas sustancias.

Considerando los mecanismos de acción molecular de la metoclopramida y los metabolitos presentes en el J atropha curcas $L$, es posible que el antagonismo que se presentó se deba a un incremento en la producción de acetilcolina (Ach), por la interacción de los receptores serotoninergicos 5-HT4 con la metoclopramida y el efecto anticolinesterasa de la planta, hecho que conllevaría a un agonismo de los receptores nicotínicos de los ganglios simpáticos y de la medula suprarrenal, lo que ocasionaría una mayor producción de catecolaminas. Estas actuarían sobre receptores adrenérgicos beta-3 y beta-2 a nivel intestinal (22-24) lo que conllevaría a un efecto inhibitorio sobre la motilidad intestinal. (Figura 1)

Este posible aumento en las concentraciones de Ach, conllevaría a que esta actué a nivel de los receptores M3 a nivel gastro-pilórico, lo que ocasionaría una contracción tónica a nivel pilórico, lo que dificultaría el vaciado gástrico y retrasaría el efecto pro cinético de las sustancias problema. Así como un posible agonismo del receptor $\mathrm{M} 1$ gástrico y duodenal, que estimularía una mayor secreción de acido clorhídrico y reducción de bicarbonato respectivamente, esto sumado a la actividad antitripsina del Jatropha curcas L., condicionaría una mayor secreción de colecistoquinina. Esta última, tendría un efecto inhibitorio del vaciamiento gástrico (25-28).

Se plantea la posibilidad de que la Ach actúe a nivel de los receptores $M 1$, en neuronas mientéricas no adrenérgicas y no colinérgicas a nivel de duodeno, yeyuno e íleon. Lo que llevaría a una mayor liberación de GABA y ATP (29-33), esto sumado con el efecto regulatorio de la secreción de óxido nítrico de la planta (34)y la presencia de atherospermidine en la misma (35-37), inducirían un estado de relajación del musculo liso intestinal lo que contribuiría a la disminución de tránsito intestinal. 


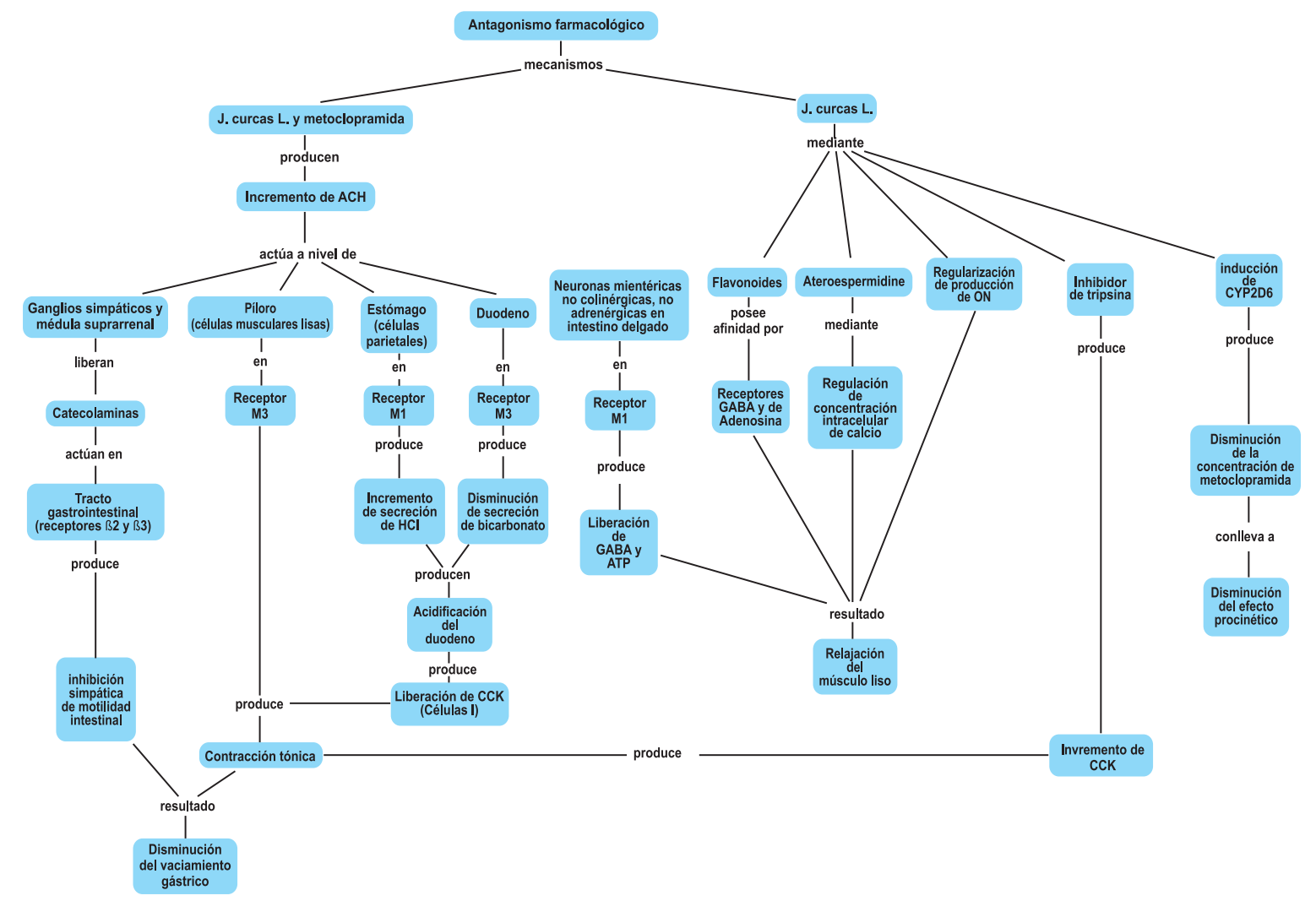

Figura 1. Posibles mecanismos de interacción farmacológica antagónica entre J.curcas L. y metoclopramida. Ach: acetilcolina, HCl: ácido clorhídrico, CCK: colecistoquinina, GABA: ácido gamma-aminobutírico, ON: óxido nítrico.

Estudios de los flavonoides y alcaloides de otras plantas medicinales, evidencian actividad a nivel del sistema de biotransformación de xenobioticos, esto llevaría a plantear como hipótesis el posible efecto inductor del J atropha curcas L. en el citocromo P450, en especial en la CYP2D6, isoforma responsable de la biotransformación de la metoclopramida $(38,39)$. Lo que ocasionaría un metabolismo acelerado de la metoclopramida a nivel hepático, lo cual explicaría la disminución del efecto pro cinético de la interacción.

La importancia de los resultados presentados radica en que en algunas regiones del Perú y Latinoamérica, el consumo del piñón blanco se da como medicina tradicional, como purgante. Del mismo modo los centros de atención primaria en dichas zonas optan por un esquema terapéutico a base de metoclopramida con el fin de acelerar el transito intestinal. Si extrapolamos los resultados obtenidos, a un posible uso simultaneo de ambas sustancias en humanos, no se al canzaría la respuesta terapéutica esperada, dado el efecto antagónico de la interacción.

Si bien, no se alcanzaría el efecto purgativo de ambas sustancias, esta interacción podría acarrear consecuencias fatales para la salud, dado que podría agravar cuadros de constipación $(40,41)$. Lo que conllevaría al acumulo de gases tóxicos, como el amonio, el que en altas concentraciones Ilevaría a un cuadro de encefalopatía hepática portosistémica $(42,43)$.

En conclusión, se evidenció un antagonismo farmacológico sobre la motilidad intestinal, entre el extracto etanólico de la semilla de J curcas L. y metoclopramida, que se explicaría por la interacción entre el sistema colinérgico, adrenérgico, GABAérgico y de neuropéptidos sobre la glándula suprarrenal, sistema nervioso central y gastrointestinal. 


\section{AGRADECIMIENTOS}

Por su apoyo y colaboración: al Dr. Frank Lizarazo Caparó, Decano de la Facultad de Medicina Humana de la USMP, al Dr. Benjamín Castañeda Castañeda, Director del Instituto de Investigación de la FMHUSMP, Profesores Angel Alvarado Yarasca y Berta Loja Herrera del Centro de Investigación de Medicina Tradicional y Farmacología de la FMHUSMP, y al Técnico Carlos Pante Medica, Bioterio de la FMH-USMP.

\section{Fuente de Financiamiento}

El presente trabajo fue financiado a través de recursos propios de los investigadores.

\section{Conflictos de interés}

Los autores declaran no tener conflictos de interés en la publicación de este artículo.

\section{REFERENCIAS BIBLIOGRÁFICAS}

1. López Sáez, J., Pérez Soto, J. Potencial etnomedicinal de dos especies tropicales del género Jatropha L. Medicina Naturista. 2011; 5(1): 8-12.

2. Mejia K, Rengifo E. Plantas medicinales de uso popular en la amazonia peruana. Agencia Española De Cooperación Internacional $(\mathrm{AECl})$ e Instituto De Investigaciones De La Amazonía Peruana (IIAP), Lima, Perú. 2000.

3. Odusote $O$, Abioye A, Rotibi M. Jatrophacurcas L. seed oil Linn (Euphorbiaceae): contraceptive activity and an oral formulation. Nig Qt J Hosp Med 2002; 12(1-4): 44-47.

4. Osoniyi O, Onajobi F. Coagulant and anticoagulant activities in JatrophacurcasL.latex. Journal of Ethnopharmacology Noviembre 2003; 89(1): 101-105

5. Goel G, Makkar HP, Francis G, Becker K. Phorbol esters: structure, biological activity, and toxicity in animals. Int J Toxicol 2007; 26(4):279-88.

6. Li CY, Devappa RK, Liu JX, Lv JM, Makkar HP, Becker K. Toxicity of $J$ atropha curcas phorbol esters in mice. Food ChemToxicol 2010; 48(2):620-5

7. Devappa RK, Rajesh SK, Kumar V, Makkar HP, Becker K. Activities of J atropha curcas phorbol esters in various bioassays. Ecotoxicol Environ Saf 2012;78:57-62.

8. Ficha técnica de Jatropha curcas. [internet]. [Consultado 2012 jul 29]. Disponible en: http://www.elsitioagricola.com/articulos/ cultivosEnergeticos/ J atrophaCurcas_FichaTecnica.pdf
9. Tomás-Guilléna E, Farriols-Danésa A, Cantarell-Aixendrib C Juárez-Giméneza JC, Interacciones entre plantas medicinales y fármacos inmunodepresore, MedClin [revista en internet], 2006 [Consultado 2012 jul 29]; 127(5):177-84.

10. Zavala Flores E, et al. Dosis-respuesta sobre la motilidad intestinal y el sistema nervioso de la interacción entre J atropha curcas $L$ y metoclopramida. Acta Méd Peruana 2013; 30(3):120-127.

11. Tres J.C.. Interacción entre fármacos y plantas medicinales. Anales Sis San Navarra [revista en la Internet]. 2006 Ago [citado 2012 Nov 24] ; 29(2): 233-252. Disponible en: http://scielo.isciii.es/scielo. php?script $=$ sci_arttext\&pid=\$1137-66272006000300007\&Ing=es.

12. Cerrate E. Manera de preparar plantas para un herbario. Lima: Museo de Historia Natural, Universidad Nacional Mayor de San Marcos; 1969.

13. Eduardo Lazcano Ponce, Eduardo Salazar•MarUnez, et al. Ensayos clínicos aleatorizados: variantes, métodos de aleatorización, análisis, consideraciones éticas y regulación. rev salud pública de México [citado 2012 oct 31]; 2004 (6)001.46, disponible en: http:// www.facmed.unam. mx/ deptos/ salud/ censenanza/ spiii/ spiii/ ensayos. pdf

14. Handbook of Laboratory Animal Science. Essential Principles and Practices. $2^{\text {a }}$ ed. Vol. 1. Boca Raton FL: CRC Press; 2003.

15. Fernando Tato, Bases metodológicas del ensayo clínico. [libro en internet] Santiago de Chile: servicio de publicaciones universidad de Santiago de Compostela, campus universitario sur; 1998 [acceso 03 noviembre 2012]. en: http://books. google.com. pe /books?id=2MqhqpldnoUC\&pg=PA40\&dq=\%E2\%8 $0 \% 9$ Cdoble+ciego\%E2\%80\%9D+de+los+ensayos+cl\%C3\%ADnicos, \&hl=es\&sa $=$ X\&ei $=$ LCSVUMylJ4b28gSpvlHoCg\&sqi $=2 \& v e d=0 C C o Q-$ 6AEWAA\#v $=$ onepage\& $q=\% E 2 \% 80 \% 9$ Cdoble $\% 20$ ciego\%E2\%80\%9D\%20 de\%20los\%20ensayos\%20cl\%C3\%ADnicos\%2C\&f =false

16. Reglamento del comité de ética para el uso de animales de investigación. [Interrnet] [citado 22/10/2012]. Disponible en: http:// www.ins. gob. pe/ repositorioaps/ 0/2/ jer/ ciea_cronograma/ REGLAMENTO_Y_RJ_CIEA.pdf

17. Feitosa CM, Freitas RM, Luz NNN, Bezerra MZB, Trevisan MTS. Acetylcholinesterase inhibition by somes promising Brazilian medicinal plants. Braz J Biol 2011; 71(3): 783-789.

18. Matsuda T, Ago Y, Takuma K. Pharmacological profiles of galantamine: the involvement of muscarinic receptor. Nihon Shinkei Seishin Yakurigaku Zasshi 2012 Feb;32(1):1-8.

19. Tinco, A., Arroyo, J., Bonilla B. Efecto del extracto metanólico de J atropha macrantha Müll Arg, en la disfunción eréctil inducida en ratas. An Fac Med 2011; 72(3): 161-8

20. Li C, Devappa R, et al. Toxicity of J atrophacurcasphorbol esters in mice. Food Chem Toxicol 2010; 48(2): 620-625.

21. Goodman y Gilman. Bases Farmacológicas de la Terapéutica. Cantabria: Editorial McGraw Hill; 2007.

22. Anthony A, Schepelmann S, Guillaume JL, Strosberg AD, Dhillon AP, Pounder RE, et al. Localization of the beta(beta)3-adrenoceptor in the human gastrointestinal tract: an immunohistochemical study. Aliment Pharmacol Ther. 1998;12:519-525.

23. Seiler R, Rickenbacher A, Shaw S, Balsiger BM. alpha- and betaadrenergic receptor mechanisms in spontaneous contractile activity of rat ileal longitudinal smooth muscle. J Gastrointest Surg 2005; 9:227-235. 
24. Coman OA, Păunescu H, Ghiță I, Coman L, Bădărăru A, Fulga I. Beta 3 adrenergic receptors: molecular, histological, functional and pharmacological approaches. Rom J Morphol Embryol. 2009; 50(2):169-79.

25. Chen YF, Chey WY, Chang TM, Lee KY. Duodenal acidification releases cholecystokinin. Am J Physiol. 1985; 249(1):29-33.

26. Hormonas-Neuropéptidos gastrointestinales. [internet]. [Consultado 2012 jul 29]. Disponible en: http://www.hvil. sld.cu/bvs/archivos/250_hormonas\%20neuropeptidos\%20 gastrointestinales\%20.pdf

27. Säfsten B. Duodenal bicarbonate secretion and mucosal protection. Neurohumoral influence and transport mechanisms. Acta Physiol Scand Suppl. 1993;613:1-43.

28. Micheletti R, Schiavone A, Giachetti A. Muscarinic M1 receptors stimulate a nonadrenergicnoncholinergic inhibitory pathway in the isolated rat duodenum. J Pharmacol ExpTher 1988;244(2):680-4.

29. Hamrouni AM, Gudka N, Broadley KJ. Investigation of the mechanism for the relaxation of rat duodenum mediated via MI muscarinic receptors. Auton Autacoid Pharmacol 2006 ;26(3):27584.

30. Zizzo MG, Mulè F, Serio R. Functional evidence for GABA as modulator of the contractility of the longitudinal muscle in mouse duodenum: role of $\mathrm{GABA}(\mathrm{A})$ and $\mathrm{GABA}(\mathrm{C})$ receptors. Neuropharmacology 2007;52(8):1685-90.

31. Rotondo $A$, Serio $R$, Mulè $F$. Functional evidence for different roles of GABAA and GABAB receptors in modulating mouse gastric tone. Neuropharmacology 2010; 58(7):1033-7.

32. Pabón Ludy C, Hernández-Rodríguez Patricia. Importancia química de J atropha curcas y sus aplicaciones biológicas, farmacológicas e industriales. Rev Cubana PlantMed [revista en la Internet]. 2012 Jun [citado 2012 Oct 09] ; 17(2): 194-209. Disponible en: http://scielo.sld.cu/scielo.php?script=sci_arttext\&pid=S1028$47962012000200008 \& \operatorname{lng}=\mathrm{es}$.

33. EhsanOskoueian, NorhaniAbdullah, WanZuhainisSaad, Abdul Rahman Omar, Syahida Ahmad, WenBinKuan, et al. Antioxidant, anti-inflammatory and anticancer activities of methanolic extracts from Jatrophacurcas Linn. J ournal of Medicinal Plants Research 2011; 5(1): 49-57.

34. Dipankar Das Gupta, Enamul Haque, Nahidul Islam, Shafiqur Rahman, Mahbub Hasan, Baigid Alam Shibib. Alkaloid and Steroid from the Stem Bark of Jatropha curcas (Euphorbiaceae). Dhaka Univ J Pharm Sci 2011; 10(1): 9-11.

35. Cortes D, Torrero MY, Pilar D’Ocon M, Candenas L, Cavé A, Had AH. Norstephalagine and atherospermidine: two smooth muscle relaxant aporphines from Artabotrys maingayi. J Nat Prod. 1990 Mar-Apr;53(2):503-508.

36. Ivorra MD, Martinez F, Serrano A, D'Ocon P. Different mechanism of relaxation induced by aporphine alkaloids in rat uterus. J Pharm Pharmacol 1993 May;45(5):439-443.

37. Desta Z, Wu GM, Morocho AM, Flockhart DA. The gastroprokinetic and antiemetic drug metoclopramide is a substrate and inhibitor of cytochrome P450 2D6. Drug Metab Dispos 2002;30:336-43.

38. Perwitasari DA, Wessels JA, Van der Straaten RJ, Baak-Pablo RF, Mustofa M, Hakimi M, Nortier JW, Gelderblom H, Guchelaar HJ. Association of $A B C B 1,5-H T 3 B$ receptor and CYP2D6 genetic polymorphisms with ondansetron and metoclopramide antiemetic response in Indonesian cancer patients treated with highly emetogenic chemotherapy. J pn J Clin Oncol 2011 Oct; 41(10):1168-76

39. Kř́žžková J, Burdová K, Stiborová M, Křen V, Hodek P. The effects of selected flavonoids on cytochromes P450 in rat liver and small intestine. InterdiscipToxicol 2009 Sep; 2(3):201-4.

40. Seltzer R. Evaluation and diagnosis of constipation. GastroenterolNurs. 2012; 35(5):343-8.

41. Campollo O. Mac Gillivray B. Mc Intyre Neil. Association of plasma ammonia and GABA levels and the degree of hepatic encephalopathy. Rev Invest Clín 1992; 44(4):483-90.

42. Rodrigo R, Cauli O, Gomez-Pinedo U, Agusti A, Hernandez-Rabaza V, Garcia-Verdugo JM, Felipo V. Hyperammonemia induces neuroinflammation that contributes to cognitive impairment in rats with hepatic encephalopathy. Gastroenterology 2010; 139(2):675-84.

43. J. Aguilar Reina. Encefalopatía hepática. Medicine. 2012; 11(11):652-9

\section{Correspondencia:}

Alberto Salazar Granara

Dirección: Av. Alameda del Corregidor 1531-

Las Viñas, La Molina. Lima - Perú.

Teléfono: 943576001.

Correo electrónico: alberto.salazar@gmail.com
Recibido: 03 de Enero de 2014 Aprobado: 20 de Mayo de 2014 